\title{
Quality 4.0. Time of revolutionary changes in the QMS
}

\author{
Dmitry Yurin ${ }^{1, *}$, Antonina Deniskina ${ }^{1}$, Boris Boytsov ${ }^{1}$, and Miron Karpovich ${ }^{2}$ \\ ${ }^{1}$ Moscow Aviation Institute (National Research University), Volokolamskoe shosse, 4, Moscow, \\ 125993, Russia \\ ${ }^{2}$ Voronezh State Technical University, 20 years of October st., 84, Voronezh, 394006, Russia
}

\begin{abstract}
The article examines the tendencies and prospects for constructing adaptive mechanisms for the functioning of active systems, taking into account the influence of the tendencies of the fourth industrial revolution, called "Industry 4.0", on the quality management system of enterprises. The main components of "Industry $4.0 "$ is the global digitalization of all enterprise processes, including management processes, which makes the process of creating learning mechanisms of functioning (MFF) of active systems, which are able to improve their functioning over time, considered relevant. The described construction of the GFM is based on the use of learning processes carried out using probabilistic iterative (recurrent) algorithms. These algorithms make it possible, as a result of processing current information, to make up for the lack of a priori data and, ultimately, to achieve the best, from a certain point of view, performance indicators.
\end{abstract}

\section{Introduction}

Quality 4.0 is a term used by quality professionals in the era of the fourth industrial revolution, also known as Industry 4.0. This article discusses new opportunities and prospects for the development of the quality system of industrial enterprises in the context of the use of new industrial cyber-physical technologies.

Disruptive technologies were the driver of the first, second and third industrial revolutions. Each revolution has resulted in a significant increase in productivity.

The first industrial revolution began in the 18th century, when mechanization and steam engines significantly increased production productivity. Developments such as spinning machines and steam power have ensured that technology, not labor, determines which countries will be the world's production leaders. At this stage of development, the quality system of industrial enterprises is limited only by the quality control of the goods produced by the workers and the consumer. The emphasis is on the volume or level of production rather than the quality of each product. Control does not focus on reducing costs or eliminating waste, waste and inefficiency, but simply getting the job done. Working conditions are not important, the productivity of each worker is simply maximized.

\footnotetext{
* Corresponding author: yurinds@rambler.ru
} 
Electricity led to the second industrial revolution in the 19th century. During this revolution, mass production and assembly lines, conveyors were launched. Mass production provided significant increases in factory productivity, but made self-control less effective. The workers did not have time to control quality with such labor productivity. As a result, the quality system focuses on adherence to standards that are produced to a minimum level of quality (eg acceptable quality level). A financial measure of quality is scrap and processing of non-compliant products.

The third industrial revolution began in the middle of the 20th century. Productivity is enhanced by the use of computers and information and communication technologies. Increased productivity leads to less human participation in the workplace (eg headcount shrinks. Autonomous robotic systems are replacing manual labor. Quality becomes a business imperative. The emphasis is on meeting customer requirements and increasing customer satisfaction. Continuous improvement to gain competitive advantage for by reducing quality costs, waste and inefficiency in end-to-end production cycles Productivity is increased by stabilizing processes at a high level Efficiency, standardization of work and involvement of all workers in activities that create quality.

The prospects for the onset of the fourth industrial revolution caused by a new stage in the development of technologies were actively discussed in 2016-2017. For the first time, the term Industry 4.0 (Industrie 4.0) was introduced in 2011 when developing a strategy for the development of Germany and provided for the transition to fully automated digital production controlled by intelligent systems in real time in constant interaction with the external environment, going beyond the boundaries of one enterprise, with the prospect of unification to the global industrial network of Things and services. In general, Industry 4.0 technologies include: Internet of Things (IIoT); artificial intelligence, machine learning and robotics; cloud computing; big data (Big Data, Data Science); additive manufacturing; integration system; modeling; augmented and virtual reality.

Analysis of the above components of Industry 4.0 allows us to conclude that there is currently a tendency to move to active enterprise management systems that widely use artificial intelligence technologies. Considering that in active systems the goals and objectives of its elements can change in the process of functioning, along with the tasks of adaptive control, it is also necessary to solve the problems of adaptive identification.

Let us consider in more detail the trends and prospects of constructing adaptive mechanisms for the functioning of active systems.

With adaptive identification and control in active systems, the current information received by the Center from system elements during the control process is used to change the parameters of the functioning mechanism (planning procedures, incentives, etc.) and achieve the goal of the system as a whole [1-6]. In this case, the most natural seems to be the use of the theory and technology of adaptation and training, developed initially in relation to the problems of control of automatic systems [7-10]. In particular, the corresponding apparatus is traditionally used in the construction of procedures for analysis, planning and forecasting in economic systems: in [11], for example, an adaptive approach in economic and mathematical modeling, retrospective analysis and forecasting of the economic development of various countries is considered, in [12] - an adaptive linear dynamic model of the inputoutput balance used for calculations in the automated system of planned calculations.

However, in contrast to the well-known formulations of adaptation and learning problems in automatic systems, with adaptive control in hierarchical organizational (socio-economic) systems, it is necessary to take into account the "human factor" associated with the presence of elements of their own goals. At the same time, the far-sighted active element (AE) of the quality management system can predict the control actions of the Center and choose its states so that, influencing the results of analysis, identification and management, maximize its own target function. Thus, it turns out that the Center is forced to work under conditions of 
uncertainty, and the available a priori information is so small that there is no way to design an effective deterministic mechanism in advance. The latter makes it relevant to create learning mechanisms of functioning (MFF) of active systems that are able to improve their functioning over time.

\section{Experimental}

The construction of GFM is based on the use of learning processes carried out using probabilistic iterative (recurrent) algorithms [13]. These algorithms make it possible, as a result of processing current information, to make up for the lack of a priori data and, ultimately, to achieve the best, from a certain point of view, performance indicators. The idea of progressiveness lies at the heart of the GFM construction. Its essence is as follows. Let the state of the AE observed by the Center in the period $t$ be characterized by the value $y_{t}$, and $y_{t} \leq p_{t}$, where $p_{t}$ is the true state, or the potential of the AE. Consider the approximation of the potential: $p_{t}=W_{t}(c)$, where $\mathrm{c}$ is some parameter unknown to the Center, and $W_{t}-$ is a monotonically increasing function. To obtain an estimate of the unknown AE parameter, we will use some well-known training procedure:

$$
\mathrm{c}_{t}=I_{t}\left(c_{t-1}, y_{t}\right), t=0,1, \ldots ; c_{-1}=c_{0}
$$

We will assume that, in the absence of activity $y_{t}=p_{t}$, this procedure converges in some probability-theoretic sense to some optimal estimate of the parameter $-c^{*}$ :

$$
\mathrm{c}_{t}=I_{t}\left(c_{t-1}, p_{t}\right) \rightarrow c^{*}, t=0,1, \ldots
$$

Let us now assume that in AMF the planning and stimulation of the AE is based on the current estimates of the parameter $-\mathrm{c}_{t}$ :

$$
x_{t+1}=\pi_{t}\left(c_{t}\right) ; \varphi_{t}=f_{t}\left(x_{t}, y_{t}\right) ; x_{0}=x^{0}
$$

where $x_{t}$ are plans and $\varphi$ are incentives at period t. The problem is that the AE chooses its state $y_{t}$ from the set of solutions of the game $R_{t}\left(\sum_{t}, p_{t}\right)$, that maximize its own objective function $w_{t}\left(\varphi_{t}, \ldots, \varphi_{t+T}\right)$ for the given mechanism of functioning $\sum_{t}=\left(I_{t}, \pi_{t}, f_{t}\right)$ and potential pt. Since the state $y_{t}$, generally speaking, differs from the true one - $\mathrm{p}_{t}$, the estimate (1) is not consistent.

On the other hand, for an accurate estimate of the parameters in accordance with expression (2), it is sufficient that $y_{t}=p_{t}, t=0,1, \ldots$ In turn, for this equality to be satisfied, it is sufficient that the gain of the AE, obtained as a result of the game, increases with an increase in the state of the element. This property of the mechanism of functioning is called progressiveness by analogy with progressive systems of remuneration in industry.

Let us now turn to the consideration of the most important classes of learners of the mechanisms of functioning of active systems that have the property of progressiveness. Let us first consider learning mechanisms of functioning with decision rules based on the theory of statistical decisions [14]. Many management tasks in hierarchical organizational and economic systems are reduced to the identification or classification by the Center of the observed situations and events. As examples, one can point to the identification of "weak points" in production and the adoption of certain measures to eliminate them, determination of the winners in the competition, etc. The classification of the observed situations is carried out by one or another identification system on the basis of some decision rule. Depending on the result of the classification, management (planning, incentives) is selected. The need for training arises if a priori information is small. Ignorance in this case is overcome by training. Typically, two types of training are distinguished: pattern recognition training ("supervised") 
and classification training (self-training).

\section{Evaluation}

The structure of an active system with training in pattern recognition.

(AE) is a control object, Center (C) acts as a teacher, and a control system (SU) is a learning one. A similar situation is typical, in particular, for the process of introducing new subsystems of the ACS designed to automate some of the Center's functions (while after the end of training, the ACS functions in an autonomous mode, relieving the Center of routine operations).

Let's consider a two-alternative case for clarity. Let $\mathrm{p}$ be a random variable (AE potential) belonging with the conditional distribution density $q(k \mid p)=q_{k}(p)$ and the prior probability $Q_{k}$ to one of the two previously unknown classes $P_{k}^{0}, k=1,2, P_{1}^{0} \cup P_{2}^{0}=P$ is compact.

Let $\left\{P_{1}, P_{2}\right\}$ denote some partition of the set $P$ into two subsets,

$P_{1} \cup P_{2}=P$, and through $w_{k m}$ - the losses arising when the situation of the class $P_{1}^{0}$ is assigned to the class $P_{m}^{0}$ (or, otherwise, when the situation of the class $P_{k}^{0}$ falls into the subset $P_{m}$ ).

As shown in [15], all the known decision rules by the Center for pattern recognition follow from the general decision rule: $p \in P_{1}$ if $\mu_{12}(p)<0 ; p \in P_{2} ; p \in P_{2}, \mu_{12}(p) \geq 0$, where $\mu_{12}(p)=\left(w_{11}-w_{12}\right) Q_{1} q_{1}(p)+\left(w_{21}-w_{22}\right) Q_{2} q_{2}(p)$

We will approximate $\mu_{12}(p)$ in the SD as follows:

$$
\hat{\mu}_{12}(c, p)=p-c
$$

where $\mathrm{c}$ is an adjustable CS parameter. Solving the problem:

$\psi(c)=\int_{P}\left[\mu_{12}(p)-\hat{\mu}_{12}(c, p)\right]^{2} d p \longrightarrow$ min and considering the sequence $p_{t}, \mathrm{t}=0,1, .$. uncorrelated, it is easy to obtain a converging (under the known conditions) learning algorithm for the EA in the form $c_{t}=I_{t}^{0}\left(c_{t-1}, p_{t}\right) \longrightarrow{ }_{t} c^{*}=\arg \min _{c} \psi(c)$;

$$
\begin{aligned}
I_{t}^{0}\left(c_{t-1}, p_{t}\right)==\left\{\begin{array}{l}
c_{t-1}\left(1-\gamma_{t}\right)+\gamma_{t}\left(\omega_{12}-\omega_{11}+h\right) / l, p_{t} \varepsilon P_{1}^{0} \\
c_{t-1}\left(1-\gamma_{t}\right)+\gamma_{t}\left(\omega_{22}-\omega_{21}+h\right) / l, p_{t} \varepsilon P_{2}^{0}
\end{array}\right. \\
\mathrm{t}=0,1, \ldots ; h=\int_{P} p d p ; l=\int_{P} d p .
\end{aligned}
$$

Note that this assumes the presence of instructions from the teacher - Center about which class belongs to the observed situation $\mathrm{p}_{\mathrm{t}}$.

Let the control actions (plans, stimuli) in the GMF with pattern recognition are formed on the basis of the approximation of the decision rule (4) and the current parameter estimates obtained using the procedure (5):

$$
c_{t}=I_{t}^{0}\left(c_{t-1}, y_{t}\right)
$$

where $y_{t}$ are the AE states observed by the Center, not necessarily coinciding with its potential $\left(y_{t} \leq p_{t}\right)$. It is assumed that the Center (teacher) can establish to which class $\left(P_{1}^{0}\right.$ 
or $P_{2}^{0}$ ) an arbitrary situation $y_{t}$ belongs.

However, the value of the potential $p_{t}$ is unknown to him (otherwise the problem is reduced to pattern recognition in the absence of activity). At the same time, the Center is not able to identify cases of incomplete use of an element of its potential, i.e. situations $y_{t}$, in which $y_{t} \leq p_{t}$. . We assume that the potential of the AE in the period $\tau, \tau=\overline{0, T}$ is a random function with a bounded distribution density: $q\left(p_{t}\right) \leq q^{*}, p_{T} \in P$. At the same time, at the moment of choosing the state $\mathrm{y}_{-} \mathrm{t}$ of the $\mathrm{AE}$, the current value of the potential $\mathrm{p}$ and the density of the potential distribution for the entire period of foresight $q\left(p_{t}\right), \tau=\overline{t+1, T}$, are known. form:

The mathematical expectation of the objective function of the AE in the period thas the

$$
\begin{gathered}
\omega\left(\varphi_{t}, \ldots, \varphi_{t+T}\right)=f\left(x_{t}, y_{t}\right)++\sum_{\tau=t+1}^{t+T} \rho_{\tau-t} \int_{P} d p_{t+1} q\left(p_{t+1}\right) \ldots \int_{P} d p_{\tau} q\left(p_{\tau}\right) f\left(x_{\tau}, y_{\tau}\right), \\
1>p \geq p_{t+1}>0, \tau=\overline{t+1, t+T}
\end{gathered}
$$

The hypothesis of the programmed behavior of the $\mathrm{AE}$ is considered [16]: in each period, the element first draws up a program for the entire period of foresight, and then chooses its state based on this program. The set of possible states of the AE in the period $t$ is $R_{t}\left(\sum_{t}, p_{t}\right)=$ $\left\{y_{t}^{*} \mid \omega\left(f\left(x_{t}, y_{t}^{*}\right), \ldots, f\left(x_{t+T}, y_{t+T}^{*}\right)\right) \geq \omega\left(x_{t}, z_{t}\right), \ldots, f\left(x_{t+T}, z_{t+T}\right)\right), z_{t} \leq p_{t}, \tau=$ $\left.\overline{t, t+T}, p_{\tau} \in P\right\}$.

The AE's benevolence towards the Center is assumed: $R_{t}\left(\sum_{t}, p_{t}\right) \ni p_{t}$, then the AE chooses the state $y_{t}^{*}=p_{t}$. In other words, $\mathrm{AE}$ does not underestimate its state if it is not beneficial for it.

We will say that there is training in pattern recognition in an active system if the parameter estimates obtained on the basis of the Center's observations (6) converge to the optimal estimate $c^{*}$. We denote:

$$
\begin{gathered}
\theta(y-x)=\left\{\begin{array}{l}
1, y \geq x ; \\
0, y<x,
\end{array} \omega \equiv \sum_{k, m=1}^{2}(-1)^{k+m} \omega_{k m},\right. \\
\delta(y-x)=\left\{\begin{array}{l}
0, y \neq x ; \\
\infty, y=x, \int_{P} \delta(y-x) f(y) d y=f(x) .
\end{array}\right.
\end{gathered}
$$

Then the following theorem is valid: for learning to recognize patterns in an active system based on the learning procedure (6), it is sufficient that procedure (5) takes place.

$$
\begin{aligned}
& x_{t+1}=c_{t}, f\left(x_{t}, y_{t}\right)=\theta\left(y_{t}-x_{t}\right) \\
& y_{t} q^{*} T \omega \leq l, t=0,1, \ldots
\end{aligned}
$$

Evidence. Without loss of generality, it suffices to consider the case $t=0$. We denote $u\left(y_{0}, \ldots, y_{t}\right)=\omega\left(\varphi_{0}, \ldots, \varphi_{t}\right)$, where $\varphi_{t}=f\left(x_{t}, y_{t}\right), x_{\tau}=c_{\tau}, \tau=\overline{0, T}$. Consider an arbitrary sequence $p_{0}, \ldots, p_{t}$ and show that

$$
\Delta_{t}=u\left(y_{0}, \ldots, y_{t-1}, y_{t}, p_{t+1}, \ldots, p_{T}\right)-u\left(y_{0}, \ldots, y_{t-1}, y_{t}, p_{t+1}, \ldots, p_{T}\right) \leq 0 \text { for any } y_{\tau} \leq
$$


$P_{\tau}, 0 \leq t \leq T$. We denote $\frac{\partial \Delta_{t}}{\partial y_{t}}=\int_{P} d p_{1} q\left(p_{1}\right) \ldots \int_{P} d p_{t} q\left(p_{t}\right) \frac{\partial \mu_{t}}{\partial y_{t}}$.

Taking into account expressions (5) - (7) and conditions of theorem (8), we have:

$$
\begin{gathered}
\frac{\partial \mu_{t}}{\partial y_{t}}=p_{t} \frac{\partial f\left(x_{t}, y_{t}\right)}{\partial y_{t}}+\sum_{\tau=t+1}^{T} p_{\tau} \int_{P} d p_{t+1} q\left(p_{t+1}\right) \ldots \int_{P} d p_{\tau} q\left(p_{\tau}\right) \frac{\partial f\left(x_{\tau}, p_{\tau}\right)}{\partial x_{\tau}} \frac{\partial x_{\tau}}{\partial y_{\tau}}= \\
=p_{t} \delta\left(y_{t}-x_{t}\right)-\gamma_{t+1} \sum_{k, m}(-1)^{k+m} \omega_{k m} l^{-1} \times\left[p_{t+1}+\sum_{\tau=t+2}^{T} p_{\tau} \prod_{v=t+2}^{\tau}\left(1-\gamma_{v}\right) \times\right. \\
\left.\times \int_{P} d p_{\tau} q\left(p_{\tau}\right) \delta\left(p_{\tau}-x_{\tau}\right)\right] \delta\left(y_{t}-x_{t}\right) \equiv k_{t} \delta\left(y_{t}-x_{t}\right) .
\end{gathered}
$$

Since at $x_{\tau} \bar{\epsilon} P \delta\left(p_{\tau}-x_{\tau}\right)=0$

$$
k_{t} \geq p_{t}-\gamma_{t+1} l^{-1} \omega\left[p_{t+1}+\sum_{\tau=t+2}^{T} p_{\tau} \prod_{v=t+2}^{\tau}\left(1-\gamma_{v}\right) q\left(x_{\tau}\right)\right] \geq p_{t}\left[1-\gamma_{t+1} l^{-1} \omega q^{*}(T-t)\right] \geq 0
$$

The second inequality takes place due to $0<\gamma_{\tau}<1$,

$0<p_{v} \leq p_{\tau}<1, \tau<v, q\left(x_{\tau}\right) \leq q^{*}, \tau=\overline{0, T}$, and the third is due to the condition (8). Therefore, for any t $\Delta_{t} \leq 0$ and $\sum_{t=0}^{T} \Delta_{t}=u\left(y_{0}, \ldots, y_{t}\right)-u\left(p_{0}, \ldots, p_{t}\right) \leq 0$ for any $y_{\tau} \leq p_{\tau}$, $\tau=\overline{0, T}$. But then $R_{0}\left(\sum_{0}, p_{0}\right) \ni p_{0}$, and according to the principle of benevolence $y_{0}^{*}=p_{0}$. Similarly, for any $\mathrm{t}=1,2, \ldots y_{0}^{*}=p_{t}$. In this case, the input sequence in the procedure $(6) \mathrm{y}_{\mathrm{t}}$, $\mathrm{t}=0,1, \ldots$ uncorrelated and, due to the procedure (5), $c_{t} \longrightarrow c_{t}^{*}$, Q.E.D.

Let, for example, the interference has a Gaussian distribution $q(\xi)=N\left(0, \sigma^{2}\right)$ on a segment of length $1, l\rangle \sigma$. Then $q^{*} \cong \frac{1}{\sqrt{2 \pi} \sigma}$ and according to the condition (8) should be $\omega y_{t} \leq \frac{\sqrt{2 \pi} l \sigma}{T}$. If the interference is evenly distributed over a segment of length $l$, that $q=1 / l$ and should be $\omega y_{t} \leq l^{2} / T$. Essentially, condition (8) is the condition for the progressiveness of the GMF. It defines the limitations on the learning procedure, in which a forward-looking AE does not underestimate its capabilities. The corresponding training procedures are called progressive. At the same time, it is feasible to use "good" procedures for pattern recognition in automatic systems [17].

Next, we pose the problem of identifying areas or groups using the decision rule based on the Center's self-learning. Let us introduce for each, so far unknown region $P_{k}, \mathrm{k}=1,2, \ldots$ the loss function $\mathrm{F}_{\mathrm{k}}(\mathrm{c}, \mathrm{p})$, where $\mathrm{c}$ is an unknown parameter. The average risk assessing the quality of the classification is minimized:

$$
\psi(c)=\sum_{k=1}^{2} \int_{P_{k}} F_{k}(c, p) q(p) d p \longrightarrow \rightarrow \min
$$

(here $\mathrm{q}(\mathrm{p})$ is the distribution density of the potential of the element $\mathrm{p}$ ).

We put $F_{1}(c, p)=p^{2}, F_{2}(c, p)=k(p-c)^{2}$ where $\mathrm{k}$ is a constant coefficient. It is easy to show that for $\mathrm{k} \gg 1$ the self-learning algorithm has the form:

$$
c_{t}=I_{t}^{k}\left(c_{t-1}, p_{t}\right) \equiv c_{t-1}+y_{t} \max \left(0, p_{t}-c_{t-1}\right) \longrightarrow{ }_{t} \longrightarrow c^{*}=\arg \min _{c} \psi(c) \text {. }
$$


We will say that there is a learning classification in an active system if the estimate based on the observation of the states of the $\operatorname{AE}\left(y_{t}\right)$

$$
c_{t}=I_{t}^{k}\left(c_{t-1}, y_{t}\right)
$$

converges to optimal $\left(\mathrm{c}^{*}\right)$. Obviously, AMF progressiveness is sufficient for teaching classification. Consider, for example, an AMF with the following planning and incentive procedures (3):

$$
x_{t}=c_{t-1}, \varphi_{t}=a y_{t}-b x_{t}
$$

Using an approach similar to that described in the previous section, we obtain one of the sufficient conditions for progressiveness - the so-called condition of p-guaranteed progressiveness [4] - in the following form:

$$
a \geq b p \gamma_{t}
$$

Note that it includes the parameters of training and stimulation procedures. Thus, to learn the classification, it is enough to restrict ourselves to the choice of suitable stimulation procedures (11), (12) and tuning (10).

The result obtained has a simple interpretation. Let the governing body of enterprises elements of the system - act as the Center. The center should single out two groups of enterprises - lagging $(\mathrm{k}=1)$ and leading ones $(\mathrm{k}=2)$ - solving the problem of risk minimization (9). Then, estimate (10) can be used as a plan if the incentive procedure $\mathrm{f}$ is chosen appropriately so that condition (12) is satisfied. If such a choice is not possible, then it is necessary to reduce $\gamma_{t}$ the gain so that condition (12) is still satisfied. Note that if the plan $y_{t}<x_{t}=c_{t-1}$ is not fulfilled, the tasks for the next period $x_{t+1}=c_{t}$ according to expressions (10) and (11) do not change, and if they are overfulfilled, they increase. This corresponds to the widely used principle of planning "from achieved" [4].

From the progressiveness condition (12), it follows that for the implementation of OMP, corresponding incentive costs are required. Therefore, it is of interest to formulate the problems of constructing incentive procedures that minimize such costs for given training and planning procedures,

$$
\varphi_{t}=f\left(x_{t}, y_{t}\right) \longrightarrow \underset{f}{\min }
$$

The corresponding GMF will be called minimally progressive. It is easy to see that for the minimum progressiveness of the above-considered GMF (10), (11) with (or), it is $a>0$ (or $b>0$ ) sufficient that equality holds in condition (12), i.e.

$$
a=b p \gamma_{t}
$$

The problem of identifying active systems is to determine their structure and parameters from observations. It arises when studying the properties and characteristics of an active element for the purpose of its subsequent control, i.e. when creating adaptive mechanisms, in which, based on the identification of an object, optimal control actions (plans, controls, incentives) are developed. This identification process involves two independent procedures, namely structural identification and parameter identification. If the laws governing the behavior of the system are known, then its structure is set a priori; otherwise, pattern recognition and classification algorithms are successfully used. When solving problems of 
identifying parameters in active systems, the main attention is paid to ensuring the progressiveness of AMF [4]. Since the solution of the game of elements belongs in this case to the boundaries of the sets of their possible states $\left(y_{t}=p_{t}\right)$, the feasibility of accurate identification of the constraint model (structure) of the AE is ensured when using "good" identification procedures in automatic systems.

The modern theory of identification in automatic systems provides the governing body with an extremely wide choice of customizable models, quality criteria and identification algorithms. To identify deterministic structures, we can use response analysis. In [4,5], conditions of strong progressiveness were obtained for the case of a multidimensional AE; in, a weaker sufficient condition for a scalar AE. The problem of exact identification of the $\mathrm{AE}$ structure characterized by a scalar parameter is considered. In the case of nondeterministic structures, methods with training are preferable: in, a number of conditions such as guaranteed progressiveness (12) were obtained, which ensure the identification of the nondeterministic structure of the AE. The case of a linear AE structure is considered in detail.

It is of interest to construct GFMs with the maximum possible rate of convergence of estimates (1) to the optimal value of the parameter $c^{*}$. This can be done on the basis of absolutely optimal algorithms developed in the information theory of identification [18]. This theory allows the Center, for each specific class of problems, characterized by one or another level of a priori information, to unambiguously determine the tunable model, quality criterion and identification algorithm that provide the maximum possible rate of convergence of the parameter estimates to their optimal values. Consider, for example, the simplest active identification problem, when the AE constraint model has the form:

$$
p_{t}=c^{*}+\xi_{t}
$$

Where $\xi_{t}$ is an independent interference with a distribution density $q(\xi)$ and zero mathematical expectation. The equation for the optimal tunable model is $\hat{p}_{t}=c_{t-1}$, where $c_{t-1}$ is the scalar estimate. In the case of Gaussian interference, an absolutely optimal $\left(q(\xi)=N\left(0, \sigma^{2}\right)\right)$ identification algorithm.

$$
c_{t}=c_{t-1}-\frac{1}{t}\left(c_{t-1}-p_{t}\right) \underset{t}{\longrightarrow} c^{*}
$$

Suppose that algorithm (16) is implemented in the GMF based on the observation of the AE states $y_{t} \leq p_{t}$, and the planning and stimulation procedures are based on estimates (16) and have the form (11). From formula (4.5.5) in [4], taking into account expression (13), we obtain the condition for the minimum guaranteed progressiveness of the GMF (11) and (16) in the form $a=b$ (compare with condition (14)). Thus, the sought GFM is a combination of linear planning and stimulation procedures (11) with $a=b$ an absolutely optimal identification algorithm (16).

The effectiveness of identification and management can be increased by using group (average) estimates or standards. Let us assume that the Center can simultaneously observe the states of $\mathrm{N}$ active elements $y_{1 t} \ldots, y_{N t}$, having the same (or rather close) constraint models (15). Let us construct group (average) estimates of the potentials and states of the system elements in the period $\mathrm{t}$ :

$$
p_{t}=\frac{1}{N} \sum_{t=1}^{N} p_{i t}, y_{t}=\frac{1}{N} \sum_{i=1}^{N} y_{i t}
$$


The variance of the average potential pt is $\mathrm{N}$ times less than the variance of the potential of an individual element. Therefore, the group estimate of the average potential obtained using procedure (16) converges much faster than the estimate of the individual potential of an independent element.

Consider a GMF with planning and stimulation procedures based on group estimates of states (17) and an identification algorithm (16):

$$
\begin{gathered}
c_{t}=c_{t-1}-\frac{1}{t}\left(c_{t-1}-y_{t}\right), x_{i t}=c_{t}, \\
\varphi_{i t}=\lambda x_{i t}-y_{i t}
\end{gathered}
$$

A necessary and sufficient condition for the guaranteed progressiveness of this GMF is, according to [4], $\lambda \leq N$ The result obtained allows for a simple meaningful interpretation [4].

Let $p_{i t}$ and $y_{i t}$ be, respectively, the minimum and actual cost of production during the period, is the profit received by the i- $t\left(y_{i t} \geq p_{i t}\right), \varphi_{i t}=\lambda c_{t}-y_{i t}$ th AE per unit of production, $\lambda$ is the pricing standard. Then the GMF (18) includes an adaptive pricing procedure based on group (averaged) estimates of the production cost (15) and providing an accurate identification of the cost under the condition $\lambda \leq \mathrm{N}$. In the case of the simplest and very popular pricing principle $\lambda=1+\alpha$, where $\alpha$ is the profitability standard, $0<\alpha<1$, in a system with more than one element (no "monopoly effect"), the GMP provides an accurate identification of the cost (15) for $\lambda<\mathrm{N}$. Note that such identification is not possible in a system with one element [17-18].

\section{Conclusions}

Thus, the construction of progressive adaptive mechanisms of functioning is aimed at creating a system of methods, algorithms and programs for analysis, pattern recognition, classification, forecasting the state (potential) of production and economic elements with a high degree of approximation, decision-making, management, planning the activities of elements based on such information. taking into account their inherent activity.

To solve this problem, hierarchical human-machine control systems with such intellectual capabilities as multilevel learning and decision-making under conditions of fuzzy or highquality commands are needed. In this regard, learning mechanisms can be considered as a class of mechanisms for the functioning of organizational systems designed to reproduce simple "behavioral" functions of a person in a control system, i.e. consider them as a special subclass of mechanisms of functioning with artificial intelligence, or, in short, intelligent mechanisms (IMF). An important principle of building an IMF is its hierarchy. It is due to the need to use decision-making systems of varying degrees of complexity, capable of using less and less defined and precise commands. At the bottom level should be the teaching mechanism, at the top - the decision maker. An intermediate position is occupied by a linguistic device (translator) that implements the interaction of the decision-maker with the OMP based on the corresponding information language. The closest prototype of the IMF in the class of GMF can be considered the mechanism of functioning with pattern recognition, considered above.

In conclusion, we note that within the framework of the considered tasks of synthesizing the adaptive mechanism of functioning, it becomes possible to comprehensively consider such types of preplanned activities as analysis, identification and forecast, in conjunction with the decision-making and planning processes, the subsequent implementation of the 
planned task and stimulation. It should be emphasized that the most advanced applied models of optimal planning in organizational and economic systems are developing in the direction of taking into account the activity of their elements [19-20].

\section{References}

1. I. Potsebneva, V. Belousov, I. Fateeva, V. Lukinov, T. Folomeeva, Algorithms for hierarchical mixing of experts' opinions on the synthesis of information systems for city development management. MATEC 2018 conference network 170.01120 Doi: 10.1051 / matecconf / 201817001120

2. V. E. Mager, V. E. Belousov, E. N. Desyatirikova, S. A. Ivanov, I. V. Potsebneva, Algorithm of information processing when creating an optimal structure of a selfadjusting technical system in terms of quality parameters. 2017 Proceedings of the 2nd 2017 IEEE International Conference on Control in Technical Systems, CTS 2017 8109503, 114-117 (2017) DOI: 10.1109 / CTSYS.2017.8109503

3. A. Korchagin, A. Deniskina, I. Fateeva, Lean and energy efficient manufacturing based on the Internet of Things (IOT) in the aviation industry E3S 2019 conference network 110.02124 DOI: 10.1051 / e3sconf / 201911002124

4. Organizational optimization of construction processes through robotization. Makovetskaya E., Deniskina A., Krylov E., Urumova F. Conference network E3S 2019 9102036 DOI: 10.1051 / e3sconf / 20199102036

5. Y. Deniskin, A. Deniskina, I. Potsebneva, S. Revunova, Application of complex information objects in sectoral control systems. E3S Conference Network, 164, 10042 (2020)

6. E. N. Desyatirikova, L. P. Myshovskaya, V. I. Lutin, V. E. Mager, Yu. V. Khripunov, Algorithmization of the evaluation of solutions by the Neumann-Pearson criterion. Proceedings of the 23rd International Conference on Soft Computing and Measurements, SCM 2020 9198783, 178-181 (2020)

7. E. N. Desyatirikova, A. Osama, V. E. Mager, L. V. Chernenkaya, A. S. Akhmad, Improving the efficiency of booking systems through data mining Lecture notes 2020 on networks and systems, 95, 413-421 (2020)

8. A. V. Loginova, E. K. Algazinov, V. V. Garshina, E. N. Desyatirikova, A. V. Smolyaninov, Cooperation with employers in the field of IT training Materials of the 6th forum IEEE 2017 "Strategic partnership between universities and enterprises of hightech industries (Science. Education. Innovation)", State Unitary Enterprise 2017 2018January, 88-91 (2017)

9. L. V. Chernenkaya, E. N. Desyatirikova, V. E. Belousov, S. I. Sergeeva, N. V. Slinkova, Optimal planning of distributed control systems with active elements Proceedings of the 2nd IEEE 2017 International Conference on Control in Technical Systems, CTS 2017 8109482, 37-40 (2017)

10. V. E. Mager, V. E. Belousov, E. N. Desyatirikova, T. B. Kharitonova, D. V. Shevchenko, 2 Modeling the inverse problem of knowledge synthesis for a flexible production system Proceedings of the conference of young researchers in electrical engineering and electronics IEEE 2018, ElConRus 2018-January, 1117-1120 (2018)

11. E. Surkova, A. Klonitskaya, E. Ermolaeva, Modeling of business processes of complex organizational systems E3S Web of Conferences, 164, 10040 (2020) 
12. A. S. Kamaletdinov, A. N. Litvinov, M. V. Danilina, E. V. Surkova, A. V. Sorokina, Management of companies in the digital economy. International Journal of Economic Research, 14(15), 9-13 (2017)

13. K. Kulakov, S. Belyaeva, O. Belyantseva, A. Gamisonia, Sustainable economic development at the meso-level: factors and ratings MATEC 2018 conference network, 170, 01118 (2018)

14. K. Gumba, S. Svetlana, S. Belyaeva, S. Revunova, Methodology of innovative control of changes in business, taking into account communication aspects. MATEC Conference Network, 106, 08023 (2017)

15. S. A. Barkalov, V. N. Burkov, A. M. Khodunov, Z. M. Daulbaeva, Yu. S. Akinina, Mechanisms for stimulating initiative projects Journal of Physics 2020: Conference Series, 1679(4), 042015 (2020)

16. S. A. Barkalov, V. E. Belousov, K. A. Nizhegorodov, A. I. Polovinkina, Dichotomous decomposition of criteria for assessing complex organizational and technical systems Proceedings 2020 - 2nd International Conference on Control Systems, Mathematical Modeling, Automation and Energy Efficiency, SUMMA 2020, 9280757, 333-336 (2020)

17. S. A. Barkalov, P. N. Kurochka, N. Yu. Kalinina, A. I. Polovinkina, The model of the formation of the degree of influence of the counterparty when making management decisions in mechanical engineering. IOP 2020 Conference Series: Materials Science and Engineering, 862(4), 042045 (2020)

18. S. Barkalov, P. Kurochka, A. Khodunov, N. Kalinina, Model of choice of technology of work on the basis of multicriteria estimates. E3S 2020 Webcast, 164, 08030 (2020)

19. T. A. Averina, S. A. Barkalov, O. L. Smolyanova, Resource allocation models in the anti-crisis management program Materials of the 12th International Conference 2019 \& amp; quot; Large-scale systems development management \& amp; quot; MLSD 8911048 (2019)

20. S. A. Barkalov, S. I. Moiseev, A. M. Khodunov, New approaches to dynamic assessment of quality indicators IOP 2019 Conference Series: Materials Science and Engineering, 537(4), 042048 (2019) 